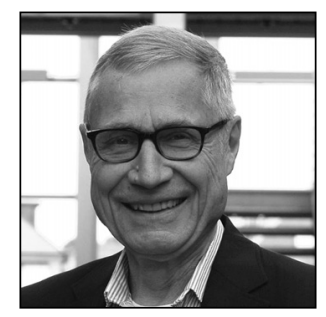

Holger Schwemer

Dr., Rechtsanwalt, Hamburg

\title{
Reformentwurf für das estnische Verwaltungsrecht (1997-1999)
}

Zur Vorbereitung seiner Mitgliedschaft in der Europäischen Union begann Estland 1996 mit seinen Vorarbeiten, das allgemeine Verwaltungsrecht und das Gefahrenabwehrrecht auf rechtsstaatlicher Grundlage zu entwickeln und an die Rechtssysteme der Mitgliedsstaaten anzupassen. Das Projekt wurde von der Europäischen Union durch finanzielle und personelle Hilfe unterstützt. Zwischen der EU und der Regierung Estlands bestand Einigkeit, dass die folgenden Ziele für die Reform unabdingbar waren:

- Rechtsstaatliche Ausrichtung des Reformentwurfs, verbunden mit der Garantie eines umfassenden Rechtsschutzes zur Wahrnehmung der subjektiven Rechte der Betroffenen.

- Einfache unüberschaubare rechtliche Ausgestaltung der rechtlichen Systeme, um die Handhabung der Gesetze bei Gerichten und Verwaltung von Anfang an sicherzustellen.

- Gewährleistung von Transparenz und Akzeptanz in demokratischer Verantwortung gegenüber dem Bürger.

Im Januar 1997 legten Prof. Dr. Ulrich Ramsauer (Richter am Oberverwaltungsgericht in Hamburg) und Prof. Dr. Holger Schwemer (Fachanwalt für Verwaltungsrecht) dem Justizministerium eine erste konzeptionelle Übersicht über das geplante Regelungswerk vor.

\section{Rahmenbedingungen}

Die angestrebten Regelungen hatten strenge Vorgaben zu beachten, die durch die sehr freiheitlich formulierte neue Verfassung von Estland vorgegeben waren. Für die Umsetzung der danach erforderlichen Regelungen war von Anfang an mit erheblichen Konflikten zu rechnen, weil es an jeglicher Kongruenz zwischen der neuen liberalstaatlichen Verfassung und den gewachsenen Verwaltungsstrukturen fehlte. Die damalige Verwaltung war sowjetisch geprägt. Die Gesetze waren generalklauselartig und damit konturenlos formuliert, die Rechtsanwendung hatte sich vom Gesetzeswortlaut weit entfernt. Das Rechtsschutzsystem selbst war im damaligen Zeitpunkt noch im Aufbau.

Die neue Verfassung von Estland ${ }^{* 1}$ (Verf.) enthält in $\S \S 8 f f$. einen umfangreichen Katalog von Grundrechten. Allerdings können die Grundrechte durch oder aufgrund eines (einfachen) Gesetzes eingeschränkt werden, wobei der einschränkende Gesetzgeber - je nach dem betroffenen Grundrecht - unterschiedlich strenge Anforderungen beachten muss. Einige Grundrechte enthalten - bedingt durch die historischen Erfahrungen - besonders weit gehende Anforderungen an das einschränkende Gesetz. Angesichts des

Eesti Vabariigi põhiseadus. - RT 1992, 26, 349. 
umfassenden Grundrechtsschutzes stellte die Verfassung somit hohe Anforderungen an den Gesetzgeber bei seinem Anliegen, funktionsfähige Verwaltungsstrukturen einzuführen. Eine effektive Verwaltung kommt ohne Grundrechtseingriffe nicht aus. Der Gesetzgeber muss somit das Verwaltungshandeln an Voraussetzungen binden, die den Grundrechtsschutz sicherstellen und trotzdem die Funktionsfähigkeit staatlicher Einrichtungen gewährleisten.

Durch § 15 Verf. wird der Rechtsweg garantiert, wenn jemand in seinen Rechten verletzt wird. § 149 der Verf. geht dabei von einem grundsätzlich mehrinstanzlichen Rechtsschutzsystem aus. Die verfassungsrechtlichen Vorgaben sind nicht auf den Fall begrenzt, dass sich der umfassende, mehrinstanzliche Rechtsschutz lediglich auf die Abwehr von Eingriffen in die in der Verf. geregelte Freiheitssphäre beschränkt. Wortlaut und Systematik zwingen zu der Auslegung, dass damit alle subjektiven Rechte - insbesondere auch Leistungsrechte auf der Ebene des einfachen Rechts - gemeint sind. Die Nichterfüllung von Leistungs- und Teilhaberechten nimmt somit an der umfassenden Rechtsschutzgarantie teil. Schließlich gilt der Schutz des § 15 Verf. und damit die Garantie des Rechtsschutzes unabhängig von der Qualität staatlichen Handelns: Die Rechtsschutzgarantie greift, wenn durch die Verwaltung Rechte verletzt oder vorenthalten werden - sei es durch zielgerichtetes oder faktisches belastendes Verwaltungshandeln, sei es durch die Nichtvornahme eines Verwaltungshandelns, auf das der Bürger einen Anspruch hat.

Der Schutz subjektiver Rechte im Verwaltungsrechtsverhältnis und ihre gerichtliche Durchsetzbarkeit waren somit aufgrund der verfassungsrechtlichen Vorgaben für Rechtsakte und für nichtregelndes Verwaltungshandeln gleichermaßen auszugestalten. Eine Einschränkung der Reformgesetze auf bestimmte Handlungsformen der Verwaltung, etwa nur auf den Verwaltungsakt, wie dies in Deutschland nach Inkrafttreten des Grundgesetzes ${ }^{*}$ mangels entsprechender Gesetze lange Jahre der Fall war, verbot sich unter diesen Umständen. Durch die für Estland geplante umfassende Konzeption des Verwaltungsrechtsschutzes werden Rechtsschutzdefizite vermieden, die in der Bundesrepublik bis Anfang der Sechzigerjahre des letzten Jahrhunderts zu erheblichen Frustrationen geführt hatten.

Dabei war aber auch zu berücksichtigen, dass nur begrenzte personelle und finanzielle Ressourcen für die Ausgestaltung des Verwaltungsrechtsschutzes zur Verfügung stehen. Estland ist ein Staat, der ca. 1,3 Mio. Einwohner hat. Bei dieser Zahl musste in Rechnung gestellt werden, dass die Anzahl der Richter und der Amtswalter, die eine rechtsstaatliche Anwendung des sich entwickelnden Verwaltungsrechts sicherstellen können, gering ist. Erschwerend trat in jener Zeit hinzu, dass die Aufgaben und Zuständigkeiten der Behörden ohne eindeutige Abgrenzungen auf eine Fülle von Behörden einschließlich der Kreise als unterer Verwaltungsebene konturenlos verteilt waren. Das Recht zur Gefahrenabwehr lag umfassend bei der Vollzugspolizei, nicht bei den Fachbehörden. Gesetzlich geregelt war in Anlehnung an das sowjetische System vor allem das repressive Recht der Polizei zur Verfolgung von Straftaten und Ordnungswidrigkeiten. Hervorzuheben ist schließlich, dass es in Estland damals wie heute im kommunalen Bereich das System der Selbstverwaltung gibt. Es bestanden damals 254 Gemeinden und Städte, deren Einwohnerzahl zwischen 100 und 0,5 Mio. lag. Die traditionelle Aufgabenwahrnehmung und Stellung der funktionalen und der kommunalen Selbstverwaltung war im Reformentwurf zu berücksichtigen.

\section{Ziel der Neuregelungen}

Anliegen der Neuregelung war somit eine rechtsstaatliche und transparente Ausgestaltung des Verwaltungsrechtsverhältnisses unter gleichzeitiger Berücksichtigung der begrenzten Ressourcen in personeller und materieller Hinsicht. Das Anliegen nach Vereinfachung des Verwaltungsrechts war dabei zwingend. Allerdings war zu beachten, dass unter der gebotenen Vereinfachung die verfassungsrechtlichen Verfahrensgarantien ebenso wenig wie die Verständlichkeit und damit die Akzeptanz der Regelungen leiden durften.

Auf der Ebene des Verwaltungsverfahrensrechts sollte dies erreicht werden durch eine eindeutige Vorgabe der behördlichen Handlungsformen, mit denen die Verwaltung verbindlich Recht setzen kann. In das Verfahrensrecht waren aber auch die sonstigen Handlungsformen der Verwaltung aufzunehmen. Bestandteil des Verfahrensrechts sollten auch grundsätzliche Aussagen über Rechtmäßigkeit und Wirksamkeit behördlichen Handelns sein.

2 Grundgesetz für die Bundesrepublik Deutschland vom 23. Mai 1949. - Bundesgesetzblatt 1949 I S.1. 
Im materiellen Recht - und dies traf in besonderer Weise auf das Recht der Gefahrenabwehr zu - waren Befugnisnormen zu formulieren, die von ihren Voraussetzungen und nach der geregelten Rechtsfolge so eindeutig sind, dass sie sowohl für den rechtsanwendenden Amtswalter als auch für den betroffenen Bürger transparent und kalkulierbar sind. Deshalb musste im Vordergrund des allgemeinen Gefahrenabwehrgesetzes ein übersichtlich geregeltes und entsprechend dem Grundsatz der Verhältnismäßigkeit gestuftes Handlungsinstrumentarium stehen, geknüpft an tatbestandlich eng definierte Voraussetzungen, die inhaltlich die verfassungsrechtlich gebotenen Konturierungen aufweisen.

Den rechtsstaatlichen Anforderungen entsprechend musste auch das gerichtliche Verfahren vor den Verwaltungsgerichten ausgestaltet werden. Das Verwaltungsgerichtsgesetz hatte der Vorgabe Rechnung zu tragen, dass der Bürger Eingriffe in seine subjektiven Rechte - unabhängig davon, ob die Eingriffe durch Rechtsakt oder durch nichtregelndes Verwaltungshandeln erfolgen - in angemessener Form gerichtlich geltend machen kann: Der Schutz der subjektiven Rechte ist unabhängig von der Qualität des Eingriffs ein Grundsatz, der durch die estnische Verfassung vorgegeben wird. Gleichermaßen war die Durchsetzung einfachgesetzlicher Leistungsansprüche zu Regeln.

Andererseits ging es aber auch darum, Unzuträglichkeiten zu vermeiden, die mit einer uferlosen Ausweitung des Verwaltungsrechtsschutzes verbunden sein können: Die Entwicklung in der Bundesrepublik Deutschland hatte gezeigt, dass die uneingeschränkte Eröffnung des Verwaltungsrechtsweges nach 1960 zu einer zunehmenden Komplizierung des Verwaltungsrechtsverhältnisses geführt hatte. Dies hatte zum einen seine Ursache darin, dass der Umfang der einfachgesetzlich einführbaren subjektiven Rechte stetig zunahm. Komplikationen folgten aber auch daraus, dass die Anerkennung von Schutzansprüchen, die unabhängig von der Qualität und dem Adressaten des Eingriffs gewährt werden und sich allein an der Eingriffswirkung orientieren, zu einer erheblichen Zunahme von Ansprüchen und damit von Klagen geführt hatte. Ein typisches Beispiel für diese Entwicklung sind Klagen bei Maßnahmen mit Drittwirkung, sei es etwa die Abwehr eines Genehmigungsbescheides durch einen Drittbetroffenen oder die Klage auf Einschreiten gegen einen „Störer“ im polizeirechtlichen Sinn.

Der mit der uneingeschränkten Rechtswegeröffnung einhergehenden Ausuferung des Verwaltungsrechtsschutzes ist der Gesetzgeber in der Bundesrepublik in der Vergangenheit mehrfach entgegengetreten. Die Eröffnung des mehrinstanzlichen Rechtsweges ist nur noch in Ausnahmefällen gegeben. Der Gesetzgeber hat den vorläufigen Rechtsschutz bei baurechtlichen Nachbarklagen eingeschränkt. Es gibt zahlreiche Möglichkeiten im Verwaltungsrecht des Bundes und der Länder, nach denen fehlerhaftes Verwaltungshandeln noch während des schwebenden Rechtsschutzverfahrens geheilt werden kann, so dass im Zeitpunkt der Entscheidung durch das Gericht die Klage des einzelnen schließlich dann doch erfolglos bleibt. $\mathrm{Ob}$ allerdings die Einschränkungen etwa des Instanzenzuges, die zunehmende Regelung von Ausschlussfristen und die spürbaren Begrenzungen von Rügerechten etwa in Planungsverfahren zur Effektuierung des Rechtsschutzes in der Bundesrepublik führen, ist bis heute nicht absehbar. Auf jeden Fall ist eine derartige Entwicklung mit Frustrationen beim betroffenen Staatsbürger verbunden, weil ihm etwas genommen wird, was er bis zu der einschränkenden Regelung zu seiner Rechtsschutzgarantie gezählt hat.

Die Gegenläufigkeiten zwischen Effektivität des Rechtsschutzes und garantiertem Rechtsschutzbestand sollte durch den Reformentwurf in Estland von vornherein vermieden werden. So bestand 1997 Einigkeit darüber, dass durch eine Optimierung der Aufgabenwahrnehmungen der Behörden und eine eindeutige Typisierung des Verwaltungshandelns unter gleichzeitiger Vereinfachung der Folgen des rechtswidrigen Verwaltungshandelns der angestrebte Effekt erreicht werden kann. Zusätzlich wurde darüber diskutiert, ob man zur Entlastung der Gerichte und zur Vereinfachung des Verwaltungsrechtsschutzes öffentliche Vergleichsstellen schaffen sollte mit der Folge, die Zahl der gerichtlichen Auseinandersetzungen zu minimieren.

\section{Die vorgelegten Gesetzesentwürfe}

Diese Ziele sollten mit den Reformgesetzen - einem Verwaltungsorganisationsgesetz, dem Verwaltungsverfahrensgesetz, einem Gefahrenabwehrgesetz, einem Gesetz über die Verwaltungsrechtsverhältnisse und dem Verwaltungsgerichtsgesetz verwirklicht werden. 


\section{Organisationsrecht}

Schon auf der Ebene des Organisationsrechts sollte - so der 1. Eckpunkt - sichergestellt werden, dass es zu einer Optimierung der Aufgabenzuweisungen an die verschiedenen Verwaltungsorgane kommt, die vor allem dem „außenstehenden Bürger“ gegenüber übersichtlich und transparent erscheint. Deshalb wurde im Reformentwurf ein Bedürfnis an der Einführung eines überschaubaren Fachbehördensystems erkannt. Allerdings konnte nicht für jede Fachfrage eine neue Fachbehörde geschaffen werden, vielmehr war auch eine Bündelung unterschiedlicher fachspezifischer Fragen bei nur einer Behörde vorzusehen. Für unspezifische, gleichsam unbenannte Aufgaben musste zudem dem traditionell gewachsenen Bedürfnis Rechnung getragen werden, die Kreisverwaltungen als Auffangbehörden mit derartigen Aufgaben zu betrauen.

Zunächst sollte die Entwicklung in der Weise noch offen bleiben, inwieweit solche „allgemeinen Verwaltungsaufgaben“ entweder von den Kommunen als übertragene staatliche Aufgabe erledigt werden, oder ob alle allgemeinen staatlichen Aufgaben künftig bei der Kreisverwaltung konzentriert werden sollten.

Im September 1998 wurde der Entwurf eines Landesorganisationsgesetzes (LOG) vorgelegt. Das Gesetz legte zunächst alle Verwaltungsträger fest - das Land, Kreise und Gemeinden sowie Personalkörperschaften der funktionalen Selbstverwaltung einschließlich der Wahrnehmung von Verwaltungsaufgaben durch juristische Personen des Privatrechts.

So lautet § 2 LOG (Verwaltungsträger):

(1) Träger der staatlichen Verwaltung ist das Land.

(2) Träger der örtlichen Selbstverwaltung sind die Städte und die Gemeinden.

(3) Verwaltungsträger sind alle Körperschaften und rechtsfähigen Anstalten, denen einzelne Aufgaben der öffentlichen Verwaltung durch oder aufgrund Gesetzes zugewiesen sind.

(4) Natürliche und juristische Personen des Privatrechts sind Träger öffentlicher Verwaltung, soweit ihnen Aufgaben der Daseinsvorsorge zur Durchführung nach Privatrecht zugewiesen sind. Die Übertragung von Hoheitsgewalt auf Private ist hingegen auf Einzelfälle beschränkt und setzt ein besonderes Gesetz voraus.

Das Gesetz regelte auch die innere Organisation der Verwaltungsträger einschließlich der Binnenstruktur von Behörden sowie die adäquaten Formen der Aufsicht und der Zusammenarbeit zwischen Behörden wie etwa die Bildung von Verbänden und die Grundsätze der Amtshilfe.

Das Landesorganisationsgesetz war darauf angelegt, dass die Verwaltungsaufgaben vorwiegend Fachbehörden (Sonderbehörden) zugewiesen werden sollten, die unter der Aufsicht einer obersten Staatsbehörde ihrerseits landesweit ihre jeweiligen Fachaufgaben wahrnehmen. Mit Ausnahme der Polizei und der Finanzverwaltung als gleichsam „geborenen“ Sonderbehörden wurde im Gesetz nicht weiter festgelegt, welche Sonderbehörden im Einzelnen zu bilden sind. Die Zuweisung weiterer Fachaufgaben an die jeweilige Sonderbehörde wurde vielmehr entsprechend künftigen Bedürfnissen der Regelung durch Verordnungen vorbehalten.

Soweit ein Bedürfnis zur allgemeinen Verwaltung durch ortsnahe Behörden bestand, sollten untere Landesbehörden eingerichtet werden. Die Aufgaben sollten bei den Kreisverwaltungen und den Oberbürgermeistern der kreisfreien Städte liegen, die im Wege der Organleihe insoweit für das Land tätig werden sollten. Während die Fachbehörden (Sonderbehörden) landesweit tätig werden, lagen demgegenüber die unbenannten - allgemeinen - Verwaltungsaufgaben bei örtlich dekonzentrierten (und damit ortsnahen) Verwaltungseinheiten.

Auszüge aus § 5 LOG (Sonderbehörden):

(1) Sonderbehörden sind Landesbehörden, die einer obersten Staatsbehörde unterstehen und für das ganze Land zuständig sind. Sie nehmen die Aufgaben wahr, die von gesamtstaatlicher Bedeutung sind und wegen ihrer Eigenart einer einheitlichen Durchführung bedürfen.

(2) Polizei und Finanzverwaltung sind Sonderbehörden.

Auszüge aus § 6 LOG (Untere Landesbehörden)

(1) Untere Landesbehörden sind Landesbehörden, die einer obersten Staatsbehörde unterstehen und für Teile des Landes zuständig sind.

(2) Die Aufgaben der unteren Landesbehörden liegen bei der Kreisverwaltung. In den Städten ist der Oberbürgermeister zugleich Organ des Landes und als solcher untere Landesbehörde. 
Diese Grundstruktur der Verwaltungsgliederung wurde im Recht der Gefahrenabwehr beibehalten. Deshalb folgte das damals konzipierte Gefahrenabwehrgesetz dem „Annexsystem“. Jede Fachbehörde war danach im Rahmen ihres Geschäftsbereichs gleichzeitig „Sonderordnungsbehörde“. Dies galt auch für die Kreisverwaltung hinsichtlich der von ihr wahrzunehmenden allgemeinen Verwaltungsaufgaben. Auf diese Weise wurde sichergestellt, dass die vorhandenen Fachkenntnisse der Fachbehörden zugleich für das Gefahrenabwehrrecht nutzbar gemacht werden.

Sollte es hingegen - was selten genug der Fall ist - um die Abwehr einer der Fachverwaltung nicht zuzuordnenden „unbenannten Gefahr” gehen, sollte eine möglichst ortsnahe und effektive Erledigung in Betracht kommen. Dem trug das allgemeine Gefahrenabwehrgesetz Rechnung durch die Übertragung dieser Ordnungsaufgabe ausnahmsweise auf die Vollzugspolizei.

Strukturell war die Vollzugspolizei organisationsrechtlich von der Ordnungsverwaltung zu trennen und als eigenständige Behörde auszubilden. Die Vollzugspolizei als „Fachbehörde zur Gefahrenabwehr” hatte danach die Aufgabe, in Eilfällen vorläufige Maßnahmen zur Gefahrenabwehr zu treffen. Lediglich für den Fall der Abwehr unbenannter Gefahren wurde das damit angelegte Trennungsprinzip zwischen Ordnungsverwaltung (Sonderbehörden) und Vollzugspolizei durchbrochen, indem die Vollzugspolizei - entsprechend ihrer jederzeitigen Präsenz und Ausbildung - zugleich unbenannte Ordnungsaufgaben wahrnehmen sollte.

Auszüge aus $§ 5$ Gefahrenabwehrgesetz (Behörden zur Gefahrenabwehr):

(1) Alle Verwaltungsbehörden sind im Rahmen ihres Geschäftsbereichs zuständige Behörden zur Gefahrenabwehr (Ordnungsbehörden). Soweit danach eine Zuständigkeit nicht besteht, ist die Polizei allgemeine Behörde zur Gefahrenabwehr.

(2) Erscheint die Notwendigkeit eines Tätigwerdens als unaufschiebbar, so ist die Polizei zuständig, anstelle der an sich zuständigen Behörde vorläufige Maßnahmen zu treffen. Die zuständige Verwaltungsbehörde ist von der Polizei über den Vorgang unverzüglich zu unterrichten. Die zuständige Verwaltungsbehörde kann anordnen, dass die Polizei den Vorgang abschließend behandelt.

Gegenstand des Landesorganisationsrechts ist somit die Regelung der Wahrnehmungskompetenz der Verwaltungsbehörden. Insoweit ist für das Recht der Gefahrenabwehr das LOG um die organisationsrechtlichen Aussagen des Gefahrenabwehrgesetzes zu ergänzen. Darüber hinaus sind weitere organisationsrechtliche Vorgaben im LOG enthalten: §§ 7-9 LOG befassten sich mit den Aufgaben und den Organen der Gemeinden und Städte, §§ 11-15 mit sonstigen Personalkörperschaften (Kammern als Träger der funktionalen Selbstverwaltung), §§ 16-18 mit rechtsfähigen Anstalten des öffentlichen Rechts sowie §§ 19-20 LOG mit Personen des Privatrechts, die entweder im Wege der Beleihung oder zur Wahrnehmung von einzelnen Aufgaben der Leistungsverwaltung in privatrechtlicher Form verwaltend tätig werden.

Im Interesse der demokratisch gebotenen Transparenz waren in $\S 21$ und in $\S 22$ LOG die Binnenstruktur der Behörden geregelt - als monokratisch organisierte Verwaltung, deren Maßnahmen allein dem Behördenleiter zugerechnet werden, oder als Kollegialbehörde, deren Entscheidungen grundsätzlich mit Stimmenmehrheit zu treffen sind. Die rechtsstaatlichen Bindungen der Verwaltung wurden in dem Gesetz dadurch verfestigt, dass es im 5. Teil (§§ 24-29) die Aufsicht über Behörden im Einzelnen konkretisiert und ausgestaltet.

$\mathrm{Zu}$ einem umfassenden Organisationsrecht gehört auch die Regelung der Zusammenarbeit zwischen Verwaltungsbehörden, sei es durch Amtshilfe oder durch Bildung von Zweckverbänden. Dies ist in $\S \S 30-40$ LOG geregelt worden.

\section{Verwaltungsverfahrensrecht}

Die Rechtsakte der Verwaltung müssen aus Gründen staatlicher Transparenz einer eindeutigen Typologie folgen. Dadurch werden die Behörden zu Handlungsformen angehalten, die aus der Sicht des Bürgers vorhersehbar und kalkulierbar sind. Deshalb weist das Verwaltungsverfahrensgesetz (vom Sommer 1999) nicht nur einen abschließenden Katalog von Rechtsakten auf, mit denen die Verwaltung gegenüber dem Bürger verbindlich Recht setzt - so etwa Verwaltungsakte, Planfeststellungsbeschlüsse, verwaltungsrechtliche Verträge sowie Rechtsverordnungen und Satzungen. Es bestand vielmehr auch ein Bedürfnis dafür, „sonstiges Verwaltungshandeln” zu bestimmen, und damit Organisationsakte, aufsichtsrechtliche 
Weisungen, Anweisungen innerhalb einer Behörde sowie schlicht hoheitliches Handeln, Realakte und weitere informationelle Maßnahmen.

Für die Beteiligten am Verwaltungsrechtsverhältnis sind die Rechtsakte der Verwaltung leichter zu handhaben und besser zu kalkulieren, wenn die Frage ihrer Wirksamkeit strukturell nach ein und demselben System beurteilt wird. Zustandekommen, Wirksamkeit trotz inhaltlicher Rechtswidrigkeit sowie Aufhebung sollten zumindest bei allen Einzelrechtsakten nach denselben Grundsätzen behandelt werden.

Insbesondere die Frage nach der Nichtigkeit eines Rechtsaktes als Folge seiner Rechtswidrigkeit bedurfte einer möglichst vereinfachenden Betrachtung. Deshalb folgten die Entwürfe konsequent dem Dogma, dass Rechtsvorschriften der Verwaltung im Falle ihrer Rechtswidrigkeit nichtig sind, der Verwaltungsakt hingegen sowie alle innerdienstlichen Akte trotz Rechtswidrigkeit Gültigkeit behalten. Das Verwaltungsprozessrecht wirkt einem etwaigen Rechtsschutzdefizit dadurch entgegen, dass auf Aufhebung wirksamer rechtswidriger Einzelakte geklagt werden kann.

In seinem allgemeinen Teil bestimmt das Verwaltungsverfahrensgesetz (VwVfG) seinen Anwendungsbereich, die allgemeinen Verfahrensgrundsätze wie etwa die Beteiligtenfähigkeit und die Handlungsfähigkeit im Verfahren sowie die Durchführung mündlicher Verhandlungen und die Tätigkeit in Ausschüssen (§§ 1-28 VwVfG).

Der besondere Teil des Verwaltungsverfahrensgesetzes ist den behördlichen Handlungsformen und damit den Rechtsakten und dem nichtregelnden Verwaltungshandeln gewidmet:

- $\S \S 30-59$ VwVfG Verfahren und Wirksamkeit des Verwaltungsaktes,

- \$§60-69 Planfeststellungsverfahren,

- $\$ \$ 70-79$ der verwaltungsrechtliche Vertrag,

- $\$ \S 80-89$ schlichthoheitliches Handeln und

- $\S \S 90 f f$. VwVfG Rechtsverordnung und Satzung.

Neben der jeweiligen Begriffsbestimmung befassen sich die Abschnitte vor allem mit der Wirksamkeit der behördlichen Handlungsformen. Denn auf die Wirksamkeit, nicht auf die Rechtmäßigkeit kommt es an, wenn die Frage aufgeworfen wird, ob Rechtsakte der Verwaltung verbindlich Recht setzen können. Die Frage nach der Wirksamkeit gliedert sich ihrerseits stets in drei Abschnitte: das Zustandekommen, Wirksamkeit trotz etwaiger Rechtswidrigkeit im Gegensatz zur Nichtigkeitsfolge sowie Aufhebung behördlicher Rechtsakte. Die Voraussetzungen für das Zustandekommen, die Nichtigkeitsgründe und die Aufhebung waren entsprechend der Art des Rechtsaktes unterschiedlich zu regeln.

Diese innere Systematik der Wirksamkeitslehre wird besonders deutlich beim Verwaltungsakt:

Auszüge aus $\S 40 \mathrm{VwVfG}$ (Bindungswirkung des Verwaltungsaktes):

(1) Ein wirksamer Verwaltungsakt bindet nach Maßgabe der folgenden Vorschriften den Betroffenen und die erlassende Behörde (allgemeine Bindungswirkung). Tatbestandswirkung und Feststellungswirkungen gegenüber weiteren Personen kommen dem Verwaltungsakt nur insoweit zu, als dies gesetzlich bestimmt ist.

(2) Ein Verwaltungsakt wird gegenüber demjenigen, für den er bestimmt ist, oder der von ihm betroffen wird, in dem Zeitpunkt wirksam, in dem er ihm bekannt gegeben wird. Der Verwaltungsakt wird mit dem Inhalt wirksam, mit dem er bekannt gegeben wird.

(3) Ein Verwaltungsakt bleibt wirksam, solange und soweit er nicht zurückgenommen, widerrufen, anderweitig aufgehoben oder durch Zeitablauf oder auf andere Weise erledigt ist. Die Erhebung eines Widerspruchs oder einer Anfechtungsklage hemmt die Wirksamkeit des Verwaltungsaktes nicht.

(4) Ein nichtiger Verwaltungsakt ist unwirksam.

Hervorzuheben ist auch die Regelung des schlichthoheitlichen Handelns. Eine vergleichbare Regelung etwa im deutschen Recht gibt es nicht. Darunter versteht § 8o Abs. 1 VwVfG solche öffentlich-rechtlichen Handlungsformen der Behörden, die nicht den Charakter von Verwaltungsakten, Rechtsvorschriften oder von - auf den Abschluss öffentlich-rechtlicher Verträge gerichteter - Willenserklärungen haben, die aber zur Beeinträchtigung von Rechten der Bürger führen können. § $81 \mathrm{VwVfG}$ verpflichtet zum rechtlichen Gehör, § 82 VwVfG nennt den anzuwendenden Rechtmäßigkeitsmaßstab und damit - dem Grundrechtsschutz entsprechend - den Gesetzesvorbehalt für den Fall eines Eingriffs, i.ü. den Gesetzesvorrang. § 83 VwVfG gewährt ein Recht auf Begründung, § 84 VwVfG regelt als prozessrechtliche Komplementärnorm Abwehransprüche und die Möglichkeit ihrer prozessualen Durchsetzung. 
In diesem Zusammenhang ist auf weitere Differenzierungen hinzuweisen und zu erwähnen, dass für bestimmte Handlungen ohne Regelungscharakter das VwVfG weitere Begriffsbestimmungen einschließlich besonderer Rechtmäßigkeitsvoraussetzungen nennt, so für Maßnahmen im Sonderstatusverhältnis, für öffentlich-rechtliche Willenserklärungen, für hoheitliche Realakte und für informationelle Maßnahmen (§§ 85-88 VwVfG).

Exemplarisch ist dafür etwa die Regelung über informationelle Maßnahmen. Sie lautet:

$\S 88 \mathrm{VwVfG}$ (Informationelle Maßnahmen):

(1) Behörden sind berechtigt, die Öffentlichkeit über ihre Arbeit und deren Ergebnisse zu informieren (allgemeine Öffentlichkeitsarbeit), soweit gesetzlich oder durch Weisung fachlich übergeordneter Behörden nichts anderes bestimmt ist.

(2) Warnungen, Empfehlungen und ähnliche auf die Beeinflussung des Verhaltens der Allgemeinheit gerichtete informationelle Maßnahmen dürfen von Behörden im Rahmen ihrer Zuständigkeiten nach pflichtgemäßem Ermessen vorgenommen werden. Sie müssen der Erfüllung der der Behörde übertragenen Aufgaben dienen, auf zutreffenden Tatsachen beruhen und den Grundsatz der Verhältnismäßigkeit beachten.

\section{Gefahrenabwehrrecht}

Dem Bedürfnis nach eindeutigen behördlichen Handlungsformen und übersichtlichen Aufgabenverteilungen wurde auch im Gefahrenabwehrrecht Rechnung getragen.

Kompetenziell wurde danach die Zuständigkeit zur Gefahrenabwehr - wie bereits oben erwähnt - nach einem „Annexsystem” der jeweiligen Fachbehörde als Sonderordnungsbehörde zugewiesen. Nur bei unbenannten Gefahren, also Gefahren, die sich fachlich nicht zuordnen lassen, sollte die Polizei als „allgemeine Fachbehörde zur Gefahrenabwehr" tätig werden. Die Zuständigkeit der Polizei wurde i.ü. strikt begrenzt auf ihre Eilkompetenzen für vorläufige Maßnahmen zur Gefahrenabwehr und damit auf jene Fälle, bei denen ein rechtzeitiges Einschreiten der zuständigen Fachbehörde nicht möglich ist (Eilfälle). Außerdem wurde in das Gefahrenabwehrgesetz als dem einheitlichen befugnisbegründenden Gesetz für Ordnungsverwaltung und Polizei ein Vorbehalt für die Aufgabe der Strafverfolgung durch die Polizei aufgenommen.

Insoweit ist die vollständige Fassung des bereits oben wiedergegebenen $\S 5$ Gefahrenabwehrgesetz nachzutragen

$\S 5$ Gefahrenabwehrgesetz (GefAbwG) (Behörden zur Gefahrenabwehr):

(3) Die Polizei nimmt ferner die Aufgaben wahr, die ihr durch besondere Bestimmungen zugewiesen sind. Nur soweit es sich dabei auch um Aufgaben der Gefahrenabwehr handelt, gelten die Vorschriften dieses Gesetzes ergänzend.

(4) Die Verhinderung von Straftaten durch die Polizei richtet sich nach diesem Gesetz. Soweit die Polizei zur Strafverfolgung tätig wird, gilt die Strafprozessordnung. Die Zuordnung einer polizeilichen Maßnahme beurteilt sich nach dem erkennbaren Zweck; im Zweifel gilt das Gesetz, nach dem die Maßnahme rechtmäßig wäre.

Für die Definition der Gefahr geht das Gefahrenabwehrgesetz nicht nur von der herkömmlichen „objektiv hinreichenden Wahrscheinlichkeit eines nicht unerheblichen Schadens, der einem Schutzgut der öffentlichen Sicherheit oder Ordnung in überschaubarer Zukunft droht”, aus (§ 3 Abs. 1). Vielmehr befasst es sich auf der Primärebene, d.h., wenn es um die Rechtfertigung des Eingreifens geht, auch mit der Anscheinsgefahr und dem Gefahrenverdacht. Die Regelung lautet:

$\S 3$ GefAbwG (Gefahrentatbestand):

(2) Geht es um die Rechtfertigung eines Eingreifens zur Gefahrenabwehr, so werden unter dem Vorbehalt der Verhältnismäßigkeit die Anscheinsgefahr und der Gefahrenverdacht einer Gefahr gleichgesetzt. Bei einer Anscheinsgefahr besteht im Zeitpunkt des Einschreitens der Eindruck einer Gefahr oder einer Störung. Ein Gefahrenverdacht liegt vor, wenn sich im Zeitpunkt des Einschreitens die Möglichkeit einer Gefahr nicht ausschließen lässt. 
Diese Regelung wird konsequent bei der Bestimmung des für die Gefahr Verantwortlichen fortgeführt. Neben der Verantwortlichkeit des „Handlungsstörers” und des „Zustandsstörers” kennt § 4 für die Primärebene auch den Verdachts- bzw. Anscheinsstörer:

$\S 4$ GefAbwG (Verantwortlichkeit):

(3) Der in $\S 3$ Abs. 2 geregelte Rechtsgedanke gilt für die Feststellung der Verantwortlichkeiten entsprechend.

Geht es um das Ob des Eingreifens und damit um die Primärebene, zwingt die Effektivität des Gefahrenabwehrrechts dazu, wirkliche Gefahr und Anscheinsgefahr gleich zu behandeln.

Insoweit wird allerdings den Herangezogenen ein besonderes Opfer für das Allgemeininteresse an einer effektiven Gefahrenabwehr abverlangt, weil der Frage nach den wahren Verantwortlichen angesichts der Kürze der zur Verfügung stehenden Zeit zur Rechtfertigung des Eingriffs bei „vermuteten Gefahren” nicht nachgegangen werden kann. Dem trägt das Gesetz auf der Sekundärebene jedoch Rechnung, indem es demjenigen einen Ausgleichsanspruch zubilligt, der als nach § 4 Abs. 3 Satz 1 Verantwortlicher zur Gefahrenabwehr herangezogen worden ist (§35 Abs. 1 Nr. 1 GefAbwG). So ist etwa die Sicherstellung eines Speditionsgutes rechtmäßig, wenn der Polizei verschiedene glaubwürdige Meldungen über das Speditionsgut als angebliches Gefahrengut zugegangen sind. Allerdings muss dem betroffenen Spediteur eine angemessene Entschädigung gezahlt werden, wenn sich später herausstellt, dass die angenommene Gefahr tatsächlich nicht bestand. Hinzuweisen ist darauf, dass dann, wenn lediglich eine einzige „unrichtige” Anzeige vorliegt, von einer Anscheinsgefahr nicht gesprochen werden kann, so dass dann bereits die Sicherstellung als solche rechtswidrig ist.

Das Handlungsprogramm der Gefahrenabwehrbehörden (Fachbehörden und Polizei) orientiert sich am Grundsatz der Verhältnismäßigkeit und der Effektivität:

- In erster Linie ist der für die Gefahr durch sein Verhalten oder als Sachherr Verantwortliche heranzuziehen. Ihm gegenüber sollen die Ge- oder Verbotsverfügung zur Gefahrenabwehr ergehen ( 88 GefAbwG).

- Sollte der Verantwortliche im Einzelfall zur Gefahrenabwehr nicht in der Lage oder aus sonstigen Gründen seine Inanspruchnahme untunlich sein, muss die Behörde an seiner Stelle im Wege der unmittelbaren Ausführung zur Gefahrenabwehr tätig werden können (§ 9 Abs. 1 GefAbwG). Der Verantwortlichkeit ist aber - nun auf der Sekundärebene - dadurch Rechnung zu tragen, dass der Verursacher der Gefahr kostenpflichtig für die staatlichen Vorkehrungen zur Gefahrenabwehr ist (§ 9 Abs. 2 GefAbwG).

- Nur wenn auch die Behörde keine Möglichkeiten zur Abwehr einer erheblichen Gefahr hat, also auch zur Eigenhandlung im Wege der unmittelbaren Ausführung nicht in der Lage ist, ist eine Inanspruchnahme Unbeteiligter möglich (§ 10 GefAbwG). Auf der Sekundärebene ist dann jedoch sicherzustellen, dass die Kosten letztlich vom Verantwortlichen getragen werden, wobei im Außenverhältnis zunächst der Staat in Vorleistung treten muss (§ 35 Abs. 1 Nr. 2 GefAbwG).

Das Gefahrenabwehrgesetz regelt in $§ \S 12-34$ so genannte „besondere Befugnisse” der Gefahrenabwehrbehörden. Dazu gehören die Ingewahrsamnahme einer Person, die Durchsuchung von Personen, die Durchsuchung von Sachen, Eingriffe in das Besitztum, die Sicherstellung, Platzverweisungen und Aufenthaltsverbote, Anordnungen gegenüber Versammlungen, die Datenerhebung und die Datenverarbeitung zur Gefahrenabwehr. Dieser besonderen Eingriffsermächtigungen bedurfte es, weil die betroffenen Grundrechte an das dem Grundrechtseingriff zu Grunde liegende Gesetz besondere Anforderungen stellen, die von der allgemeinen Generalermächtigung zur Gefahrenabwehr (§§ 7-10) nicht erfüllt werden. Die Bestimmungen sind aber auch deshalb erforderlich, weil sie für bestimmte Fallgruppen typisiert sind und damit dem handelnden Amtswalter in komplexen Gefahrensituationen eine Hilfe sind. Diese Bestimmungen sind zugleich leges speciales zum allgemeinen Teil des Gefahrenabwehrrechts.

Insoweit bedurfte einer besonderen Regelung:

$\S 12$ GefAbwG (Besondere Befugnisse):

(1) Soweit im folgenden Abschnitt besondere Befugnisse zur Gefahrenabwehr geregelt sind, gehen diese Bestimmung den $\S \S 7-10$ vor. Die in den $\S \S 7-10$ genannten Voraussetzungen kommen jedoch insoweit zur Anwendung, als entsprechende Aussagen in den nachfolgenden Vorschriften nicht oder nicht vollständig bestehen. 
(2) Soweit im folgenden Abschnitt einer Behörde zur Gefahrenabwehr eine besondere Befugnis verliehen ist, schließt das den Erlass von Ge-/Verbotsverfügungen ebenso wie die Befugnis zur unmittelbaren Ausführung und zum Vollzug der Maßnahmen ein, soweit dies zur Erreichung des angestrebten Zwecks erforderlich ist. Dies gilt für die Ausübung weiterer besonderer Befugnisse im Sinne dieses Abschnitts nur dann, wenn sie in der befugnisbegründenden Vorschrift im Sinne des Satz 1 ausdrücklich genannt sind. I.ü. richtet sich die Wahrnehmung einer besonderen Befugnis nach den für sie geltenden Vorschriften.

Wenn z.B. § 20 Abs. 1 Nr. 1 GefAbwG bestimmt, dass Sachen sichergestellt werden dürfen, „um eine gegenwärtige Gefahr abzuwehren“, so ermächtigt dies ohne weitere Hervorhebung in § 20 auch zum Erlass einer entsprechenden Herausgabeanordnung oder zur Vornahme einer unmittelbaren Ausführung, wenn die Polizei die Sache findet. Eingeschlossen ist auch die zwangsweise Durchsetzung der Sicherstellung, soweit dies zur Erreichung des Zwecks erforderlich ist ( 12 Abs. 2 Satz 1). Weitere flankierende Maßnahmen, etwa die Ausübung eines Betretungsrechts mit dem Ziel, überhaupt erst in den Besitz der sicherzustellen Sache zu kommen, sind durch die Befugnisnorm hingegen nicht legitimiert. Insoweit muss nach § 12 Abs. 2 Satz 3 die Ermächtigung zum Betreten zusätzlich geprüft werden, weil in der Regelung der Sicherstellung diese besondere Befugnis nicht erwähnt ist (vgl. § 12 Abs. 2 Satz 2).

\section{Gesetz über die Verwaltungsrechtsverhältnisse}

Im Oktober 1999 wurde der Entwurf des Gesetzes über die Verwaltungsrechtsverhältnisse dem Justizministerium vorgestellt. In diesem Gesetz waren die Grundbegriffe der Ansprüche des Bürgers gegen die Verwaltung auf Begünstigung und auf Abwehr belastenden Verwaltungshandelns zu regeln sowie die Fragen, woraus sich Ansprüche im Verwaltungsrecht ableiten und gegen wen sie sich richten. An sich hätten in das Gesetz auch die Staatshaftung und damit der Anspruch des einzelnen auf Entschädigung gegen die Verwaltung insbesondere bei unrechtmäßigem Verwaltungshandeln aufgenommen werden müssen. Im Justizministerium wurde jedoch damals mehrheitlich die Auffassung vertreten, Staatshaftung gehöre in das Privatrecht, zumal sie auch zum damaligen Zeitpunkt dort geregelt war. Das führte dazu, dass gleichsam nur die Primäransprüche gegen die Verwaltung im Gesetz über die Verwaltungsrechtsverhältnisse kodifiziert wurden, nicht dagegen Ansprüche auf Schadensersatz und Entschädigung. Für Letztere war zunächst die Entwicklung des Privatrechts abzuwarten.

Im Mai 2001 wurde dann doch ein Staatshaftungsgesetz verabschiedet, das im Wesentlichen für die Primärebene die Regelungen des Gesetzes über die Verwaltungsrechtsverhältnisse übernahm, und für die Sekundärebene ein System der öffentlichen Staatshaftung einführte.

In seinem Allgemeinen Teil regelt das Gesetz über die Verwaltungsrechtsverhältnisse, wer die Beteiligten der Rechtsbeziehung sind, wann ein Verwaltungshandeln öffentlich-rechtlich ist und damit dem Anwendungsbereich des Gesetzes unterfällt, welchen Bindungen die Verwaltung unterfällt (Gesetzesvorbehalt und Gesetzesvorrang) und woraus sich die Rechte des Bürgers gegen die Verwaltung auf Durchsetzung dieser Bindungen ableiten können.

Die Abgrenzung des privatrechtlichen von einem öffentlich-rechtlichen Verwaltungshandeln hat grundlegende Bedeutung für das Verwaltungsrecht, zumal die Anwendung des allgemeinen und besonderen Verwaltungsrechts davon abhängig ist, dass die Verwaltung öffentlich-rechtlich gehandelt hat. Deshalb ist auf die Abgrenzungsnorm noch einmal hinzuweisen und zugleich anzumerken, dass sie die Zweifelsregelung aufweist, dass Verwaltungshandeln beim Fehlen anderweitiger Zuordnungskriterien dem öffentlichen Recht unterfällt. Soll davon abgewichen werden, trifft gleichsam die Verwaltung die Beweislast. Dabei ist die Zuordnung von Leistungs- und Abwehrfälle unterschiedlich zu beurteilen. Schließlich sind Besonderheiten bei der Beleihung zu beachten. Dies alles findet seinen Niederschlag in der nachfolgenden Bestimmung:

$\S 3$ GesVerwRV (Öffentlich-rechtliches Verwaltungsrechtsverhältnis):

(1) Ein Rechtsverhältnis zu einer juristischen Person des öffentlichen Rechts ist öffentlich-rechtlich, sofern es nicht ausdrücklich den Regeln des Privatrechts unterstellt ist. Einzelne Aufgaben der Daseinsvorsorge können privatrechtlich ausgestaltet werden. Sofern das Gesetz nichts anderes bestimmt, sind Bedarfsdeckung und Vermögensverwaltung durch Behörden nur nach Privatrecht zulässig. 
(2) Für die Rechtsnatur eines belastenden Verwaltungshandelns entscheidet nicht, wie der Hoheitsträger hätte handeln müssen, sondern wie er tatsächlich gehandelt hat. Berechtigt oder verpflichtet eine Rechtsgrundlage einseitig einen Hoheitsträger, so ist die Rechtsanwendung öffentlich-rechtlich.

(3) Das Rechtsverhältnis zu einem Beliehenen richtet sich nach öffentlichem Recht, wenn sich der Beliehene im Einzelfall einer hoheitlichen Handlungsform bedient hat oder wenn sich der Anspruch dem Aufgabenbereich zuordnen lässt, für den die Beleihung erfolgt ist.

Bei der Regelung über die Bindungen der Verwaltung macht das Gesetz in $\S 4$ deutlich, dass zur Legitimation auch ein wirksames vorausgegangenes Verwaltungshandeln ausreichen kann, etwa ein öffentlichrechtlicher Vertrag, der durch seine Wirksamkeit die allgemeine Gesetzesbindung der Verwaltung überlagern kann. In $\S 5$ wird bestimmt, dass sich subjektive Rechte außer aus Sonderbeziehungen auch aus allen Rechtsvorschriften ableiten, die neben der Allgemeinheit zumindest auch dem Schutz des einzelnen zu dienen bestimmt sind. Das Gesetz stellt zudem klar, dass der Bürger keinen allgemeinen Gesetzesvollziehungsanspruch hat.

Schwerpunkt des Gesetzes ist die Regelung der Ansprüche auf Begünstigung und auf Abwehr belastenden Verwaltungshandelns.

Bei den Abwehransprüchen differenziert das Gesetz nach den Handlungsformen der Behörde. Beim Abwehranspruch gegen einen Verwaltungsakt gibt es unterschiedliche Regelungen für Abwehransprüche des Adressaten und für Drittbetroffene. § 11 befasst sich mit dem Anspruch auf Abwehr schlichten Verwaltungshandelns, § 12 mit dem vorbeugenden Unterlassungsanspruch und § 13 mit den Folgenbeseitigungsansprüchen.

Damit sind auf der Primärebene alle Ansprüche im Verwaltungsrechtsverhältnis umfassend ausgestaltet.

\section{Verwaltungsrechtsschutz}

Ohne Verzicht auf die verfassungsrechtliche Vorgabe des umfassenden Rechtsschutzes konnte mit der Neuregelung gleichwohl eine erhebliche Vereinfachung und damit einhergehend eine Effektuierung des Verwaltungsrechtsschutzes erreicht werden.

Einen ganz beachtlichen Entlastungseffekt versprach man sich durch die Einführung eines „öffentlichen Vergleichsverfahrens" an Stelle des herkömmlichen Widerspruchsverfahrens. Durch die Besetzung der „Vergleichsstelle” sollte sichergestellt werden, dass diese Verfahrensstufe nicht als überflüssiges Vorverfahren betrachtet wird, das „durchlaufen” werden muss, sondern tatsächlich zur Konfliktbewältigung genutzt wird. Praktisch sollte damit eine Mediation stattfinden, bevor geklagt wird. Leider wurde der Gedanke dann auch mit Blick auf die personellen Ressourcen verworfen.

Eine Vereinfachung bei den Klagearten wurde dadurch erreicht, dass die Leistungsklage zur zentralen Klageform erklärt wurde. Mit ihr soll nicht nur eine Begünstigung in Form eines nichtregelnden Verwaltungshandelns, sondern ebenso ein Verwaltungsakt eingeklagt werden können. Gegenstand der Leistungsklage ist aber auch die Abwehr eines nichtregelnden Verwaltungshandelns sowie die Verpflichtung der Verwaltung zur Folgenbeseitigung. Eine weitere Besonderheit gegenüber dem Rechtsschutzsystem in der Bundesrepublik wurde dadurch geschaffen, dass sich der vorläufige Verwaltungsrechtsschutz in Estland grundsätzlich nur nach einer besonders zu beantragenden einstweiligen Anordnung richten sollte, eine Suspendierung etwa eines Verwaltungsaktes als gesetzliche Folge des Hauptsacheverfahrens war nicht vorgesehen.

Der Anwendungsbereich der Feststellungsklage wurde entsprechend den Erfahrungen in der Bundesrepublik genauer definiert, er umfasste die Fortsetzungsfeststellungsklage ebenso wie die Normennichtigkeitsfeststellungsklage.

Nur beim Verwaltungsakt wurde damals - nach langer Diskussion - dem Bedürfnis an einer besonderen Klageart in Form der Gestaltungsklage Rechnung getragen. Eine Leistungsklage auf Aufhebung durch die Behörde wäre nach anfänglicher Einschätzung auch ausreichend gewesen. Andererseits setzt die Behörde mit dem Verwaltungsakt gegenüber dem Bürger im Einzelfall verbindlich Recht, zugleich ist der Verwaltungsakt Grundlage für die Verwaltungsvollstreckung. Dem Interesse an rückwirkender Aufhebung als Kompensation für das so geartete einseitige Vorgehen der Behörde - dies auch mit Blick auf die 
Verpflichtung zur Folgenbeseitigung - kann nur die Gestaltungsklage mit der Kassation durch das Gericht Rechnung tragen.

Ein Land von der Größe Estlands sollte sich nicht drei Instanzen in der Verwaltungsgerichtsbarkeit „leisten”. Deshalb wurde sichergestellt, dass grundsätzlich nur eine Rechtsmittelebene in Betracht kommt und die ausnahmsweise Anrufung einer weiteren Instanz an besondere Zulassungsvoraussetzungen geknüpft wird.

Im Übrigen enthält das Verwaltungsgerichtsgesetz für die Republik Estland vom 28.4.1999 eine Reihe von Regelungen, die sich auch im deutschen Recht bewährt haben: Dazu gehören die allgemeinen Verfahrensvorschriften (Dispositionsmaxime, Untersuchungsgrundsatz, Anspruch auf Akteneinsicht) ebenso wie die Bestimmungen über Beweiserhebung und die Arten der gerichtlichen Entscheidungen.

Der Reformentwurf wurde nicht 1 zu 1 vom Gesetzgeber übernommen. Das wäre auch mit dem Demokratieprinzip nicht vereinbar. Trotzdem enthält Reformentwurf grundlegende Vorgaben und Weichenstellungen, die bei den sich anschließenden Aktivitäten des Parlaments Berücksichtigung fanden. Damit hat der Reformentwurf die gestellte Aufgabe erfüllt. Die damals entwickelten rechtsstaatlich-demokratische Ideen leben in den heutigen Gesetzen fort. 